\title{
Combined Aerobic and Resistance Exercise Program Improves Task Performance in Patients With Heart Failure
}

\author{
Rebecca A. Gary, PhD, RN, M. Elaine Cress, PhD, Melinda K. Higgins, PhD, Andrew L. Smith, MD, \\ Sandra B. Dunbar, DSN, RN
}

\section{Program Overview}

Heart failure (HF) is a leading cause of cardiac disability that is anticipated to dramatically increase over the next several decades largely due to an aging population, earlier identification, and advances in the treatment of coronary artery disease (CAD). Aerobic exercise has gained increasing support over the past two decades as an adjunct nonpharmacological therapy to partially reverse the underlying peripheral and musculoskeletal alterations that accompany heart failure (HF), without inducing greater cardiovascular stress. Heart failure management has traditionally focused on curtailing disease progression but fewer trials have reported strategies to attenuate cardiac related disability. This journal-based activity describes the results of a randomized controlled study that investigated whether a combined aerobic and resistance training program improves performance of physical activities of daily living (PADLs) routinely performed by most New York Heart Association (NYHA) class II and III HF patients.

Accreditation Statement

This journal-based activity has been planned and implemented in accordance with the Essential Areas and policies of the Accreditation Council for Continuing Medical Education (ACCME) through the sponsorship of the Elsevier Office of Continuing Medical Education (EOCME). The EOCME is accredited by the ACCME to provide continuing medical education (CME) for physicians.

Credit Designation Statement

The EOCME designates this journal-based activity for a maximum of 3.0 AMA PRA Category 1 Credit $(s)^{\mathrm{TM}}$. Physicians should only claim credit commensurate with the extent of their participation in the activity. All other health care professionals completing continuing education credit for this activity will be issued a certificate of participation.

Educational Objectives

To support the attainment of knowledge, competence, and performance, the learner should be able to achieve the following objectives:

1. Describe an exercise program that combines aerobic and resistance activities for patients with New York Heart Association (NYHA) Class II or III heart failure (HF)

2. Describe the benefits and limitations of combined aerobic and resistance exercise on daily physical living activities for patients with NYHA Class II or III HF

3. Recognize that combined aerobic and resistance exercise improves health-related quality of life in patients with NYHA Class II or III HF

4. Recognize that combined aerobic and resistance exercise may reduce the risk of losing independence in patients with NYHA Class II or III HF

Planning Committe

Rebecca A. Gary PhD, RN; M. Elaine Cress, PhD; Melinda K. Higgins, PhD; Andrew L. Smith, MD; Sandra B. Dunbar DSN, RN; Leighton Chan, MD, PhD; Tania Dickson, PhD

Faculty Profiles \& Disclosure Information

As a sponsor accredited by the ACCME, it is the policy of the EOCME to require the disclosure of anyone who is in a position to control the content of an educational activity. All relevant financial relationships with any commercial interests and/or manufacturers must be disclosed to participants at the beginning of each activity. The faculty of this educational activity discloses the following:

Rebecca A. Gary, PhD, RN, FAHA

Nell Hodgson Woodruff School of Nursing

Emory University

Atlanta, GA 30322

Disclosures: Has nothing to disclose.

M. Elaine Cress, PhD

Department of Kinesiology

Department of Health Promotion \& Behavior

University of Georgia

Athens, GA 30602-6554

Disclosures: Has nothing to disclose.

Melinda K. Higgins, PhD

Nell Hodgson Woodruff School of Nursing

Emory University

Atlanta, GA

Disclosures: Has nothing to disclose.
Andrew L. Smith, MD

School of Medicine

Department of Cardiology

Emory University

Atlanta, GA

Disclosures: Has nothing to disclose.

Sandra B. Dunbar, DSN, RN, FAAN, FAHA

Nell Hodgson Woodruff School of Nursing

Emory University

Atlanta, GA

Disclosures: Has nothing to disclose.

Leighton Chan, MD, MPH

Deputy Editor, Archives of Physical Medicine and Rehabilitation

Bethesda, MD

Disclosures: Has nothing to disclose.

EOCME STAFF

Tania Dickson, $\mathrm{PhD}$

Disclosures: Has nothing to disclose

\section{Resolution of Conflict of Interest}

The EOCME has implemented a process to resolve conflict of interest for each CME activity In order to help ensure content objectivity, independence, and fair balance, and to ensure that the content is aligned with the interest of the public, the EOCME has resolved the conflict by external content review.

\section{Unapproved/Off-Label Use Disclosure}

The EOCME requires CME faculty to disclose to the participants:

1. When products or procedures being discussed are off-label, unlabeled, experimental, and/or investigational (not US Food and Drug Administration [FDA] approved); and

2. Any limitations on the information presented, such as data that are preliminary or that represent ongoing research, interim analyses, and/or unsupported opinion. Faculty may discuss information about pharmaceutical agents that is outside of FDA-approved labeling. This information is intended solely for CME and is not intended to promote off-label use of these medications. If you have questions, contact the medical affairs department of the manufacturer for the most recent prescribing information.

\section{Intended Audience}

This program is intended for physicians and healthcare professionals responsible for the comprehensive care for individuals with chronic illness and disabilities.

\section{METHOD OF PARTICIPATION}

In order to claim credit, participants must complete the following:

Pre-activity self-assessment questions

2. Read the activity

3. Complete the CME Test and Evaluation. Participants must achieve a score of $70 \%$ on the CME Test

Participants can complete the pre-activity self-assessment and CME Test and Evaluation online by logging-on to www.elsevierocme.com/910472-3. Upon successful completion of the online tests and evaluation form, you can instantly download and print your certificate of credit.

To better define and meet the CME needs of health care professionals and enhance future CME activities, the EOCME will conduct an outcomes-measurement survey following the conclusion of the program. This follow-up survey is designed to measure changes to participants " practice behaviors as a result of their participation in this CME activity. You will be contacted by email 60 days following the conclusion of this activity with an outcomes measurement survey. We would greatly appreciate your participation.

CME Inquiries

For all CME certificate inquiries, please contact Sandy Breslow at elsevierCME@elsevier.com. Release Date of Activity:

Expiration Date of Activity for

AMA PRA Credit: ............................... August 31, 2012

Estimated Time to Complete This Activity $\ldots \ldots \ldots \ldots \ldots \ldots \ldots \ldots \ldots$. 3.0 hour 
ABSTRACT. Gary RA, Cress ME, Higgins MK, Smith AL, Dunbar SB. Combined aerobic and resistance exercise program improves task performance in patients with heart failure. Arch Phys Med Rehabil 2011;92:1371-81.

Objectives: To assess the effects of a home-based aerobic and resistance training program on the physical function of adults with New York Heart Association (NYHA) class II and III patients and systolic heart failure (HF).

Design: Randomized controlled trial.

Setting: Home based.

Participants: Stable patients $(\mathrm{N}=24$; mean age, $60 \pm 10 \mathrm{y}$; left ventricular ejection fraction, $25 \% \pm 9 \% ; 50 \%$ white; $50 \%$ women) with New York Heart Association (NYHA) classes II and III (NYHA class III, 58\%) systolic heart failure (HF).

Intervention: A 12-week progressive home-based program of moderate-intensity aerobic and resistance exercise. Attention control wait list participants performed light stretching and flexibility exercises.

Main Outcome Measures: A 10-item performance-based physical function measure, the Continuous Scale Physical Functional Performance test (CS-PFP10), was the major outcome variable and included specific physical activities measured in time to complete a task, weight carried during a task, and distance walked. Other measures included muscle strength, HRQOL (Minnesota Living With Heart Failure Questionnaire, Epworth Sleepiness Scale), functional capacity (Duke Activity Status Index), and disease severity (brain natriuretic peptide) levels.

Results: After the exercise intervention, 9 of 10 specific task activities were performed more rapidly, with increased weight carried by exercise participants compared with the attention control wait list group. Exercise participants also showed significant improvements in CS-PFP10 total score $(P<.025)$, upper and lower muscle strength, and HRQOL $(P<.001)$ compared with the attention control wait list group. Adherence rates were $83 \%$ and $99 \%$ for the aerobic and resistance training, respectively.

Conclusions: Patients with stable HF who participate in a moderate-intensity combined aerobic and resistance exercise program may improve performance of routine physical activities of daily living by using a home-based exercise approach. Performance-based measures such as the CS-PFP10 may provide additional insights into physical function in patients with HF that more commonly used exercise tests may not identify. Early detection of subtle changes that may signal declining physical function that are amenable to intervention potentially may slow further loss of function in this patient population.

Key Words: Exercise; Functional performance measures; Health-related quality of life; Heart failure; Rehabilitation.

(C) 2011 by the American Congress of Rehabilitation Medicine

From the Nell Hodgson Woodruff School of Nursing (Gary, Higgins, Dunbar); and Department of Cardiology, School of Medicine (Smith), Emory University, Atlanta, GA; and Departments of Kinesiology and Health Promotion and Behavior, University of Georgia, Athens, GA (Cress).

Supported in part by the Clinical and Translational Science Award program (grant no. PHS Grant UL1 RR025008), the National Institutes of Health, the National Center for Research Resources; and Emory Heart and Vascular Center.

No commercial party having a direct financial interest in the results of the research supporting this article has or will confer a benefit on the authors or on any organization with which the authors are associated.

Reprint requests to Rebecca A. Gary, PhD, RN, 1520 Clifton Rd NE, Atlanta, GA 30322, e-mail: ragary@emory.edu.

0003-9993/11/9209-00122\$36.00/0

doi:10.1016/j.apmr.2011.02.022
$\mathbf{H}$ EART FAILURE (HF) is a leading cause of cardiac disability that is anticipated to dramatically increase during the next several decades, largely because of an aging population, earlier identification, and advances in the treatment of coronary artery disease (CAD). ${ }^{1}$ Aerobic exercise has gained increasing support during the past 2 decades as an adjunct nonpharmacological therapy to partially reverse the underlying peripheral and musculoskeletal alterations that accompany $\mathrm{HF}$ without inducing greater cardiovascular stress. ${ }^{2-5}$ Numerous studies, including the Randomized Controlled Trial Investigating Outcomes of Exercise Training in Heart Failure, ${ }^{6}$ supported the safety, efficacy, and improved cardiovascular outcomes associated with aerobic exercise in stable patients with systolic HF. However, some studies suggested that aerobic training alone may not optimally address the loss of muscle strength, functional decline, and progressive disability that occur. ${ }^{7-9}$ Concerns that resistance exercise may increase left ventricular remodeling and worsen hemodynamic status have dampened its wider acceptance and use among clinicians as a mode of exercise for patients with HF. The most recent guidelines for exercise in patients with HF advocate moderate-intensity dynamic resistance exercise as a safe and effective training mode, and that in conjunction with aerobic exercise, it may be most effective for maximizing physical function in this patient population. ${ }^{10}$

HF management traditionally focused on curtailing disease progression, but fewer trials reported strategies to attenuate cardiac-related disability. ${ }^{11,12}$ Performance-based measures that directly assess objective ability to perform physical activities of daily living (PADLs) are not used routinely in patients with HF or cardiovascular diseases in general. Performance measures represent aspects of physical function associated with routine daily activities that are important for maintaining independence in older adults. These measures integrate multiple dimensions of health and aging, such as disease processes, nutritional status, cardiorespiratory fitness, and psychological state, and provide a global assessment of physical function. ${ }^{13-16}$ Although performance measures are used widely in comprehensive geriatric assessment, their use has been very limited in patients with cardiac disease. However, recent studies indicated that they are practical and safe to administer and provide valuable insight regarding patients with HF and other cardio-

\section{List of Abbreviations}

\begin{tabular}{ll} 
6MWT & Six-Minute Walk Test \\
BNP & brain natriuretic peptide \\
CAD & coronary artery disease \\
CS-PFP10 & 10-Item Continuous Scale Physical Functional \\
& Performance \\
DASI & Duke Activity Status Index \\
ESS & Epworth Sleepiness Scale \\
HF & heart failure \\
HR & heart rate \\
HRQOL & health-related quality of life \\
ICD & implanted cardiac defibrillator \\
LVEF & left ventricular ejection fraction \\
MLHFQ & Minnesota Living With Heart Failure \\
& Questionnaire \\
NYHA & New York Heart Association \\
PADL & physical activity of daily living \\
RPE & rate of perceived exertion \\
t2 & after 12 weeks \\
Vo ${ }_{2}$ peak & peak oxygen consumption \\
\hline
\end{tabular}


vascular diseases, who are most vulnerable for adverse events and outcomes. ${ }^{11,12,17-20}$

Previous research in patients with HF primarily used selfreport questionnaires or such single-task performance measures as the 6-Minute Walk Test (6MWT) for cardiorespiratory fitness that indirectly assess PADLs from only 1 perspective, endurance. For example, peak oxygen consumption ( $\mathrm{VO}_{2}$ peak), the criterion standard for determining cardiorespiratory fitness, consistently has had low to moderate correlation with the ability to perform routine PADLs; very few patients perform these activities at maximum-intensity level, except those with advanced $\mathrm{HF}^{21,22}$ In addition, because many household chores more often rely as heavily on strength as endurance, an instrument that incorporates measures of muscle strength may more accurately reflect the ability to perform these routine tasks. ${ }^{23,24}$ The ability to detect subtle changes in physical function may provide valuable insight into strategies that may be implemented to slow or partially reverse the disability associated with $\mathrm{HF}^{23}$

This study examined whether a combined aerobic and resistance training program improved performance of PADLs routinely performed by most patients with New York Heart Association (NYHA) classes II and III HF. The functional performance measure, the 10-item Continuous Scale Physical Functional Performance test (CS-PFP10), which has been validated against standard exercise tests $\left(\mathrm{eg}, \mathrm{VO}_{2}\right.$ peak, muscle torque, reaction time), was used to quantify physical function. ${ }^{25,26}$ The CS-PFP10 ${ }^{25}$ provides a total score and physical domain scores (Upper- [10-Item Continuous Scale Physical Functional Performance Upper-Body Strength domain] and Lower-Body Strength [10-Item Continuous Scale Physical Functional Performance Lower-Body Strength domain], flexibility, Balance and Coordination [10-Item Continuous Scale Physical Functional Performance Balance and Coordination domain], Endurance [10-Item Continuous Scale Physical Functional Performance Endurance domain]) for serially performed tasks that closely simulates PADLs, with higher scores reflecting better physical functioning. In the present study, we analyzed the time required for dressing, kitchen and household activities, and endurance walking. Additionally, we evaluated the ability to lift weighted items designed to simulate cooking and carrying groceries. We hypothesized that participants who underwent a combined aerobic and resistance exercise program would have significant improvement in speed, weight, or both for the 10 specific tasks included in the CS-PFP10 ${ }^{26}$ compared with an attention control wait list group. In addition, we evaluated changes and correlations between physical function, disease severity, health-related quality of life (HRQOL), and functional capacity in both groups from baseline to 12 weeks as secondary outcomes.

\section{METHODS}

\section{Study Design}

A randomized repeated-measures design was used to enroll and follow up subjects from baseline to 12 weeks (time 2 [t2]). After 12 weeks, t 2 measurements were obtained.

\section{Participants}

The study sample included 24 men and women between the ages of 40 and 75 years (mean \pm SD age, 60 $\pm 10 y$ ) with a diagnosis of stable NYHA class II or III systolic HF. Eligibility criteria included living within a 100-mile radius of the institution, left ventricular ejection fraction (LVEF) of $15 \%$ to $40 \%$, diagnosis of systolic HF for a minimum of 6 months, using
Table 1: CS-P FP10 Items

Low difficulty

Pot carry test: Participant will place up to $30 \mathrm{~kg}$ of sandbags in cooking pot and transfer $\sim 1.80 \mathrm{~m}$ from 1 counter to another.

Jacket test: Participant will put on a light jacket, pull the front of the jacket together, and then remove it.

Reach test: Participant will reach as high as possible and place a sponge on an adjustable wall-mounted shelf that has a maximum height of $\sim 2.5 \mathrm{~m}$.

Moderate difficulty

Floor sweep test: Participant will sweep up a $118 \mathrm{~mL}(1 / 2$ cup) of kitty litter in a $.91 \times 1.22 \mathrm{~m}$ block.

Scarves test: Participant will pick up 4 scarves, 1 at a time, from the floor.

Laundry test 1: Participant will transfer clothes and sandbags weighing $4.5 \mathrm{~kg}$ from a top-loading washer into a front-loading dryer.

Laundry test 2: Participant will unload only the clothes from the dryer into a laundry basket, which is then placed on a cabinet $.90 \mathrm{~m}$ high adjacent to the dryer.

High difficulty

Floor down/up test: Participants will start in the standing position, sit down on the padded floor, and then immediately return to standing, finishing with arms at their side.

Stair climb test: Participants will climb 1 flight of stairs, 9-11 steps 12 inches in depth and 6.5 inches high.

Grocery test: Participant will carry a comfortable amount of groceries a total of $52.3 \mathrm{~m}$, including a set of bus steps and opening door. Maximum weight allowed is $30 \mathrm{~kg}$.

6MWT: Allowing rest breaks, the participant will walk at a pace to cover the greatest distance in 6 minutes. Lap length is $35 \mathrm{~m}$, and the participant is not verbally encouraged during the task.

current cardiac medications for a minimum of 3 months, and permission to enroll in the study by an attending cardiologist in 1 of the 2 university-affiliated HF outpatient clinics. Exclusion criteria were (1) hospitalization for an acute HF exacerbation within the prior 2 months, (2) uncontrolled angina, (3) uncontrolled hypertension (blood pressure at rest $\geq 160 \mathrm{mmHg}$ systolic or $\geq 90 \mathrm{mmHg}$ diastolic), (4) dementia or a psychological disorder that would interfere with participation in exercise, and (5) contraindications to resistance exercise (ie, aneurysm, valvular disease). After receiving university-affiliated human subjects approval, potential patients were screened in 1 of the HF clinics. If eligibility criteria were met, a letter was sent to the potential candidate, and if interested, he/she was contacted by telephone to have the study explained. If participants agreed to participate in the study, they were required to provide written informed consent before initial baseline measurements were obtained. After baseline measurements were obtained, patients were randomly assigned by using a 1:1 allocation ratio to either the exercise or attention control wait list group.

\section{Instruments}

10-Item Continuous Scale Physical Functional Performance test. The CS-PFP $10^{20}$ was used to evaluate physical function (table 1). The CS-PFP10 consists of 10 tasks that are performed serially in a manner of usual function (a person's preferred manner vs a demonstrated manner). The tasks are quantified by weight, time, distance, or a combination of these and include a battery of items ranging from easy to difficult. The 10-item battery includes (1) pot carry, (2) jacket don/doff, 
(3) scarf pick up, (4) reach, (5) sweep, (6) transfer laundry from washer and dryer, (7) floor sit down and return to stand, (8) grocery carry over $53 \mathrm{~m}$ including 4 steps, (9) climb a flight of stairs, and (10) 6-minute walk (see table 1). Five domain scores (10-Item Continuous Scale Physical Functional Performance Upper-Body Strength domain, 10-Item Continuous Scale Physical Functional Performance Lower-Body Strength domain, Upper-Body Flexibility [10-Item Continuous Scale Physical Functional Performance Upper-Body Flexibility domain], 10Item Continuous Scale Physical Functional Performance Balance and Coordination domain, 10-Item Continuous Scale Physical Functional Performance Endurance domain) and a total summary score are generated, with higher scores reflecting better physical function. Scores of 48 to 59 are associated with having an increased probability of losing independence. ${ }^{27}$ The CS-PFP10 has been validated against standard exercise capacity measures $\left(\mathrm{VO}_{2}\right.$ peak, peak torque, reaction time), which may be used to gain further insight into underlying physical impairments contributing to functional limitations. ${ }^{25}$ At the conclusion of the CS-PFP10, participants were instructed to assess their rate of perceived exertion (RPE) using the 6- to 20-RPE Borg Scale. ${ }^{28}$ In addition, participants wore a heart rate monitor (Polar) ${ }^{\mathrm{a}}$ to evaluate any heart rate (HR) change occurring with each task. For additional information about the CS-PFP10, refer to the following website: www. coe.uga.edu/cs-pfp.

Six-Minute Walk Test. The 6MWT, a frequently used, reliable, and well-validated measure in patients with $\mathrm{HF}$, was used to measure functional capacity. Participants walked for 6 minutes on a level hallway $50 \mathrm{~m}$ long at baseline and again at t2. In our experience with the 6MWT, there was no significant difference in distance when 2 consecutive walks were performed on the same day or the participant had completed the CS-PFP10 before the 6MWT. ${ }^{29-31}$

Handgrip. Handgrip strength was measured with a handgrip dynamometer (Jamar) ${ }^{\mathrm{b}}$ by using a standardized protocol in both sides (dominant and nondominant) with a precision of $0.1 \mathrm{~kg}$. Participants were asked to self-adjust the dynamometer to fit comfortably with their hand size to achieve optimal performance. For the 3 serial measurements, subjects were seated with both arms resting on a table at a comfortable level with elbows flexed at a $90^{\circ}$ angle with support, with the dynamometer facing outward from the body. A warm-up session to familiarize participants with the dynamometer was conducted to choose the best adjustment. Participants were instructed by voice command to grip the dynamometer with maximum strength. During the grip strength measurements, $\mathrm{HR}$ was monitored using the HR monitor and blood pressure was measured immediately before and after for safety reasons. Three trials were performed alternately on each side, with a rest period of at least 1 to 2 minutes between trials. The mean value for each hand was used for handgrip strength. ${ }^{32}$

Upper- (forearm flexion) and lower-body muscle strength (knee extension). These were measured by using the handheld dynamometer. For forearm flexion, the person sat at a table with the elbow at a $90^{\circ}$ angle, palm up, with the dynamometer placed 2 inches above the wrist. Participants were instructed by voice command to pull upward with maximum strength against the dynamometer. For knee extension, participants were instructed to sit on a bed with knees at a $90^{\circ}$ angle to the floor. The bed was raised so that feet were 10 to 12 inches off the floor. The dynamometer was placed 2 inches above the ankle, and when prompted, participants were instructed to kick the foot outward at a $90^{\circ}$ angle. The mean force (kilograms) of 3 trials was calculated, with rest between each of the 3 contractions. Muscle strength testing was conducted by
2 data collectors for each test to ensure reliability. If a difference of $0.1 \mathrm{~kg}$ was documented for a strength measure, another reading was obtained and mean force in kilograms was calculated as the strength for that testing procedure. ${ }^{33,34}$

Health-related quality of life. HRQOL is defined as a multidimensional concept that reflects the influence of an individual's health on the ability to function in daily life, performance of social roles, and emotional status. HRQOL was measured by using a disease-specific measure ${ }^{35}$ the Minnesota Living With Heart Failure Questionnaire (MLHFQ). ${ }^{36}$ The MLHFQ is a well-established 21-item disease-specific questionnaire designed to measure perceived physical, socioeconomic, and psychological impairment in persons with HF. Scores range from 0 to 105 , with higher scores indicative of poorer HRQOL. A change in score of 5 points or more is considered clinically significant for improvement in symptom severity and HRQOL. Middel et $\mathrm{al}^{37}$ found the MLHFQ to be more sensitive to change over time than other global measures of HRQOL. Cronbach $\alpha$ reliabilities of the total MLHFQ instrument, the physical subscale, and the emotional subscale are reported at $.87, .81$, and 0.84 and consistently are greater than .70 in published reports, respectively. ${ }^{36}$

Duke Activity Status Index. The Duke Activity Status Index (DASI) is a 12-item questionnaire that measures perceived functional capacity with a score of 0 (worst) to 58.2 (best). A difference of 2 or more units on DASI score is considered clinically meaningful. ${ }^{38}$ The DASI has been validated against $\mathrm{VO}_{2}$ peak $(r=.80 ; P<.001)$ and has been used to successfully measure functional status in patients with $\mathrm{HF}^{39}$

Epworth Sleepiness Scale. The Epworth Sleepiness Scale $(E S S)^{40}$ is a well-established 8-item self-administered questionnaire that rates, on a 4-point scale $(0-3)$, the chances of dozing off in different situations. Total ESS score ranges from 0 to 24 , with higher scores reflecting more daytime sleepiness.

Brain natriuretic peptide. Brain natriuretic peptide (BNP) was measured by using a BNP test (Triage) ${ }^{\mathrm{c}}$ to measure cardiovascular stress and ventricular load. BNP level increases in response to ventricular wall stress and volume overload from HF. BNP levels correlate well with left ventricular end-diastolic pressure, pulmonary artery wedge pressure, left ventricular hypertrophy, and systolic/diastolic dysfunction. ${ }^{41}$ As BNP level increases, the ability to perform PADLs may decrease as a consequence of worsening symptom severity. ${ }^{42}$

Charlson Comorbidity Index. The Charlson Comorbidity Index ${ }^{43}$ is well established as a measure of risk for 1-year mortality attributable to comorbid conditions and was used to assess the number and severity of comorbid diseases, using the original scoring version. Participants' medical records also were reviewed to ensure the accuracy of self-reported information concerning comorbid conditions.

\section{Procedures}

Overview of intervention: exercise program. Participants performed the combined aerobic and resistance exercise program for 12 weeks. They also received individualized instruction and a demonstration of how to monitor and record HR using the HR monitor, use of the 6- to 20-point Borg RPE Scale, ${ }^{28}$ and any symptoms experienced during the walking or strength training sessions. Supervised weekly home-based sessions for 8 consecutive weeks and then every other week were conducted by a member of the research team (nurse or exercise specialist). At each home visit, the exercise prescription was adjusted, which progressively increased both walking and resistance exercise. Participants in the exercise group also received a DVD and exercise booklet as a reference to use during the unsupervised sessions. 
Overview of intervention: aerobic exercise. Progressive low- to moderate-intensity walking was used for the aerobic exercise component. Based on the 6MWT, we estimated intensity level by using the HR reserve method, ${ }^{44}$ with participants beginning at $50 \%$ intensity and progressing to $70 \%$ intensity for a minimum of 30 minutes and a maximum of 1 hour 3 times per week. Both at rest and exercise HRs are influenced by $\beta$-blockers, which are considered optimal therapy for patients with HF. ${ }^{45}$ The HR reserve method ${ }^{44}$ takes into account the patient's HR at rest, thereby decreasing the effect of $\beta$-blockade for the exercise prescription. Participants were instructed to maintain the RPE at 12 to $15 .^{28}$ Participants were supervised during only the first week of walking to better ensure safety and that self-monitoring techniques (HR, blood pressure, RPE, symptom severity) were understood. If there were no safety issues after the first session, participants walked unsupervised and performed self-monitoring independently for the rest of the intervention period. The weekly supervised sessions were used to monitor response to the resistance training protocol. However, during the weekly home visits, activity logs (step/cord calendars) were reviewed and both the walking and resistance prescriptions were adjusted accordingly.

Overview of intervention: resistance exercise. Color coded Thera-cords ${ }^{\mathrm{d}}$ were used for the resistance exercise component. The color of the cord represents different levels of resistance. All participants began with a yellow or red cord, which reflects the lowest resistance level, to familiarize them with the exercise regimen and for safety reasons. The duration of the resistance exercise sessions was approximately 1 to 1.5 hours, depending on patient tolerance, and included a 5-minute warm up (low-intensity stretching/flexibility) and a 45- to 60-minute lower- and upper-body resistance training session. Lower-body resistance training included ankle plantar flexion and dorsiflexion; knee extension and flexion; hip extension, abduction, and adduction; and leg extension. Upper-body resistance training included exercises for shoulder abduction, flexion, and rotation; elbow extension and flexion; and wrist extension and flexion. Individual progression of resistance training was monitored and adjusted when the participant was able to perform 2 sets of 12 to 15 repetitions or less than 15 on the RPE scale. Participants were asked to perform the resistance exercises 2 or 3 times per week, but not on 2 consecutive days to avoid muscle fatigue and soreness. Several patients who were unable to perform standing exercises used a sitting protocol with the same exercise routines developed by the investigators. Most participants progressed during the 12-week study period to 3 sets of 15 repetitions 2 to 3 times weekly. Theraballs $^{\mathrm{d}}$ were used to improve handgrip strength and also were progressed as strength increased. Participants were asked to perform hand exercises a minimum of 5 minutes per day in each hand and not to squeeze the ball continuously to avoid potential adverse hemodynamic responses.

Overview of intervention: exercise adherence. Exercise adherence was assessed by using step/cord calendars and pedometers. The number of daily steps walked was assessed indirectly by using the Omron HJ 112. e $^{\mathrm{e}}$ The device resets at midnight automatically; therefore, we were able to compare steps recorded by patients on the step/cord calendar with actual pedometer data to validate patient documentation. A number of studies have shown Omron pedometers to be accurate and reliable in adults and patients with $\mathrm{HF}^{45-48}$ and a more valid measurement than self-report questionnaires. ${ }^{45}$ For adherence to the resistance exercise component, patients recorded Theracord color, exercises completed, and number of repetitions performed on the step/cord calendar. Each week, the nurse/ exercise specialist recorded adherence from the previous week on the intervention log sheet for each participant by using the step/cord calendar. To be considered $100 \%$ adherent to the protocol, 3 documented walking sessions and 2 strength training sessions weekly were required.

Overview of intervention: exercise progression. The primary method of progressing exercise was based on RPEs for both aerobic and resistance exercise because most participants was prescribed $\beta$-blockers. For the walking sessions, participants were asked to write the following values on a step/cord calendar: maximum HR, RPE during walk, number of steps walked, or symptoms during the aerobic session. For resistance exercise, the participant also was asked to record Thera-cord color and number of repetitions after each session, maximum HR, RPE, and any symptoms experienced. Patients were instructed to keep the target HR within a prescribed range and RPE at less than 15 for both modes of exercise.

Overview of intervention: attention control wait list group. Control participants were provided with instruction for stretching and flexibility movements, and no additional written materials were provided. After measurements were obtained at $\mathrm{t} 2$, control participants received the same instruction and equipment and 2 supervised home visits for resistance exercises, but received no additional outcome testing. Participants in the attention control wait list group received a total of 5 to 6 home visits during the 12-week period to control for attention received by the researchers in the intervention group.

\section{Statistical Analysis}

Values in tables are presented as mean \pm SD. Baseline differences for sociodemographic and outcome measures were tested by using independent-samples $t$ tests. Paired $t$ tests were used to determine within-group changes. Analysis of covariance was used to determine group differences at $\mathrm{t} 2$ on study outcomes, adjusting for baseline scores. Cohen $d$ statistic was use to calculate effect size for the intervention. ${ }^{49}$ Pearson $r$ correlation coefficients were used to determine relationships among the key variables (not included in tables). Statistical analyses were carried out using SPSS software (Version 16.0). Significance level of $P<.05$ was used for hypotheses testing.

\section{RESULTS}

Study recruitment occurred during a 6-month period, and 615 patients were screened for study eligibility at 1 of the 2 university-affiliated HF outpatient clinics. Of this number, $463(75 \%)$ were screen failures. Common reasons for screen failure included distance from the hospital, renal failure, NYHA class I or IV, documented nonadherence to medication regimen, or psychosocial issues that would affect compliance ability. The $152(25 \%)$ eligible participants were sent a maximum of 2 letters during a 6-month period explaining the study details. Of this number, 21 participants were recruited by letter; 6 , by flyers posted in HF clinics; and 1 , by word of mouth. Because of time commitments, 3 participants dropped out before baseline measurements were obtained, and 1 participant was excluded during baseline measurements (not included in the analysis) because of uncontrolled hypertension. Twenty-four patients were randomly assigned in a 1:1 allocation sequence to either the exercise or attention control wait list group after baseline measurements. No participant dropped out of the study after baseline measurements were obtained.

At baseline, there were no group differences in sociodemographic characteristics (table 2), total and domain scores for the CS-PFP10, muscle strength, or HRQOL (table 3). However, there were a significantly greater number of insulin-dependent 
Table 2: Baseline Sociodemographic and Clinical Characteristics for Total Group, Intervention and Control Participants

\begin{tabular}{|c|c|c|c|c|}
\hline & Total $(\mathrm{N}=24)$ & Intervention $(n=12)$ & Control $(n=12)$ & $P$ \\
\hline Age $(y)$ & $60 \pm 10$ & $59 \pm 11$ & $61 \pm 10$ & 0.56 \\
\hline Sex & & & & 0.41 \\
\hline Men & $12(50)$ & $7(58)$ & $5(42)$ & \\
\hline Women & $12(50)$ & $5(42)$ & $7(58)$ & \\
\hline Ethnicity & & & & 0.10 \\
\hline Black & $11(46)$ & $3(25)$ & $8(67)$ & \\
\hline White & $12(50)$ & $8(67)$ & $4(33)$ & \\
\hline Other & $1(4)$ & $1(8)$ & & \\
\hline NYHA class & & & & 0.41 \\
\hline ॥ & $10(42)$ & $4(33)$ & $6(50)$ & \\
\hline III & $14(58)$ & $8(67)$ & $6(50)$ & \\
\hline LVEF (\%) & $25 \pm 9(n=22)$ & $23 \pm 8(n=12)$ & $27 \pm 9(n=10)$ & 0.24 \\
\hline Weight (kg) & $99 \pm 18$ & $95 \pm 19$ & $102 \pm 18$ & 0.34 \\
\hline Height $(\mathrm{cm})$ & $171 \pm 14$ & $171 \pm 16$ & $171 \pm 12$ & 0.96 \\
\hline BMI $\left(\mathrm{kg} / \mathrm{m}^{2}\right)$ & $34 \pm 7$ & $32 \pm 7$ & $36 \pm 8$ & 0.20 \\
\hline HR (beats/min) & $72 \pm 9$ & $72 \pm 9$ & $73 \pm 9$ & 0.31 \\
\hline Systolic BP (mm Hg) & $114 \pm 17$ & $109 \pm 19$ & $116 \pm 12$ & 0.18 \\
\hline Diastolic BP $(\mathrm{mm} \mathrm{Hg})$ & $72 \pm 10$ & $70 \pm 11$ & $74 \pm 12$ & 0.67 \\
\hline ICD & $19(79)$ & $11(92)$ & $8(67)$ & 0.13 \\
\hline Comorbidity score & $2.5 \pm 1(1-5)^{*}$ & $2 \pm 1$ & $3 \pm 1$ & 0.46 \\
\hline Hypertension & $12(50)$ & $4(33)$ & $8(67)$ & 0.10 \\
\hline Myocardial infarction & $11(46)$ & $5(42)$ & $6(50)$ & 0.68 \\
\hline Diabetes & $12(50)$ & $4(33)$ & $8(67)$ & 0.10 \\
\hline Depression history & $13(54)$ & $7(58)$ & $6(50)$ & 0.68 \\
\hline Dyslipidemia & $22(92)$ & $11(92)$ & $11(92)$ & 1.00 \\
\hline No. of medications & $11 \pm 3(3-16)^{*}$ & $11 \pm 3$ & $11 \pm 4$ & 0.86 \\
\hline ACEI & $13(54)$ & $4(33)$ & $9(75)$ & 0.04 \\
\hline ARB & $16(67)$ & $10(83)$ & $6(50)$ & 0.08 \\
\hline$\beta$-Blocker & $19(79)$ & $9(75)$ & $10(83)$ & 0.62 \\
\hline Diuretic & $21(87)$ & $11(92)$ & $10(83)$ & 0.54 \\
\hline Insulin & $8(33)$ & $1(8)$ & $7(58)$ & $0.01^{\dagger}$ \\
\hline Oral hypoglycemic $(n=22)$ & $7(32)$ & $3(25)$ & $4(40)$ & 0.45 \\
\hline $6 \mathrm{MWT}(\mathrm{m})$ & $335 \pm 105$ & $364 \pm 80$ & $307 \pm 121$ & 0.18 \\
\hline
\end{tabular}

NOTE. Values expressed as mean \pm SD or $n(\%)$ unless noted otherwise.

Abbreviations: ACEI, angiotensin-converting enzyme inhibitor; ARB, angiotensin receptor blocker; BMI, body mass index; BP, blood pressure. *Range.

${ }^{\dagger} P<.01$.

patients with diabetes in the attention control wait list group, as listed in table 2 . In addition, although all patients were receiving what is considered optimal medication therapy for HF according to guidelines, ${ }^{45}$ there was a significantly greater number of attention control wait list patients receiving angiotensin-converting enzyme inhibitors and a higher proportion with angiotensin receptor blockers prescribed in the intervention group. All patients were receiving 1 or the other and outcomes therefore were unlikely to be influenced by these medication differences. Most had NYHA class III (58\%; $\mathrm{n}=14)$, mean LVEF was $25 \% \pm 9 \%$, most $(79 \%$; $n=19)$ had an implanted cardiac defibrillator (ICD), and most were obese (mean body mass index, $34 \pm 7 \mathrm{~kg} / \mathrm{m}^{2}$ ). Mean comorbidity score was $2.5 \pm 1$, with diabetes the most common medical noncardiovascular disorder. Most $(75 \% ; n=18$, ) had a diagnosis of HF for more than 2 years. Only 2 participants (1 in exercise group, 1 in control group) had scores higher than the CS-PFP10 cutoff range (48-59) of having a higher probability of losing independence. At baseline, mean CS-PFP10 score was 42 \pm 16 (range, 8-76); at $\mathrm{t} 2$, mean score was $47 \pm 17$ (range, 15-78). There were no serious adverse events in any study participant, and baseline medication changes were stable throughout the study period.

\section{Exercise Intervention Group}

Participants in the exercise group improved their time in seconds compared with baseline for completing most tasks (table 4). For tasks that involved weight and time, weight and time for the grocery carry significantly increased at $\mathrm{t} 2$, which likely brought about positive clinical and lifestyle changes. For the 6MWT, distance increased from $364 \pm 80$ to $411 \pm 110 \mathrm{~m}$ $(P<.006)$. Overall CS-PFP10 score increased from $46 \pm 15$ to $56 \pm 16(P<.003)$. Domain scores for 10-Item Continuous Scale Physical Functional Performance Upper-Body Strength domain, 10-Item Continuous Scale Physical Functional Performance Upper-Body Flexibility domain, 10-Item Continuous Scale Physical Functional Performance Lower-Body Strength domain, 10-Item Continuous Scale Physical Functional Performance Balance and Coordination domain, and 10-Item Continuous Scale Physical Functional Performance Endurance domain increased significantly. Muscle strength also improved after the 12-week exercise intervention in most areas (see table 3 ). There was greater improvement in patients with NYHA class III from baseline to 2 for CS-PFP10 total scores, increasing from $37 \pm 13$ to $49 \pm 15$ compared with $62 \pm 16$ to $68 \pm 10$ in patients with NYHA class II (not shown). 
Table 3: Muscle Strength, CS-PFP10 Score, and Health Status Changes From Baseline to t2 in Intervention and Control Participants

\begin{tabular}{|c|c|c|c|c|c|c|c|c|c|c|c|c|}
\hline \multirow[b]{2}{*}{ Variable } & \multicolumn{4}{|c|}{ Control $(n=12)$} & \multicolumn{4}{|c|}{ Exercise Intervention $(n=12)$} & \multicolumn{3}{|c|}{ Between Groups } & \multirow{2}{*}{$\begin{array}{c}\begin{array}{c}\text { Post- } \\
\text { Cohen } \\
\mathrm{d}\end{array} \\
\begin{array}{c}\text { Effect } \\
\text { Size }\end{array}\end{array}$} \\
\hline & Pre & Post & Change: Post-Pre & $\begin{array}{c}\text { Paired } \\
t \text { Test } \\
(P)\end{array}$ & Pre & Post & Change: Post-Pre & $\begin{array}{c}\text { Paired } \\
t \text { Test } \\
(P)\end{array}$ & $\begin{array}{c}\text { Pre } t \\
\text { Test }(P)\end{array}$ & $\begin{array}{c}\text { Post } t \\
\text { Test }(P)\end{array}$ & $\begin{array}{c}\text { Post } \\
\text { ANCOVA }(P)\end{array}$ & \\
\hline \multicolumn{13}{|c|}{ Muscle strength } \\
\hline $\mathrm{R}$ handgrip & $28.4 \pm 10.7$ & $28.1 \pm 9.8(n=11)$ & $-0.7 \pm 5.7(n=11)$ & .702 & $28.0 \pm 9.2$ & $35.1 \pm 8.8$ & $7.1 \pm 8.9$ & .019 & .936 & .084 & .016 & 0.76 \\
\hline$L$ handgrip & $24.8 \pm 10.4$ & $25.4 \pm 10.4$ & $0.6 \pm 4.7$ & .685 & $26.6 \pm 10.5$ & $32.0 \pm 9.0$ & $5.4 \pm 9.2$ & .069 & .675 & .109 & .063 & 0.68 \\
\hline R FA flex & $24.9 \pm 8.9$ & $21.6 \pm 8.3(n=11)$ & $-2.0 \pm 4.8(n=11)$ & .189 & $23.2 \pm 7.5$ & $27.5 \pm 4.4$ & $4.3 \pm 5.0$ & .012 & .626 & .042 & .002 & 0.90 \\
\hline L FA flex & $22.3 \pm 9.3$ & $21.8 \pm 7.6$ & $-0.4 \pm 4.5$ & .755 & $23.2 \pm 6.4$ & $27.1 \pm 4.1$ & $4.0 \pm 5.0$ & .018 & .781 & .045 & .005 & 0.87 \\
\hline $\mathrm{R}$ knee ext & $31.5 \pm 10.4$ & $31.0 \pm 9.3$ & $-0.5 \pm 7.4$ & .825 & $31.3 \pm 9.5$ & $37.8 \pm 10.4$ & $6.5 \pm 8.7$ & .025 & .952 & .110 & .036 & 0.68 \\
\hline L knee ext & $31.1 \pm 12.5$ & $30.6 \pm 10.1$ & $-0.4 \pm 76.3$ & .837 & $30.8 \pm 8.7$ & $35.7 \pm 9.5$ & $4.9 \pm 8.6$ & .072 & .943 & .222 & .086 & 0.51 \\
\hline $\begin{array}{l}\text { 6MWT } \\
\text { 6MV }\end{array}$ & $306.6 \pm 121.3$ & $309.7 \pm 135.4$ & $3.1 \pm 58.1$ & .855 & $364.3 \pm 79.7$ & $410.7 \pm 91.5$ & $46.4 \pm 46.9$ & .006 & .182 & $.045^{*}$ & .074 & 0.87 \\
\hline BNP & $105.8 \pm 159.1$ & $108.4 \pm 158.9$ & $2.6 \pm 104.3$ & .934 & $184.4 \pm 151.6$ & $308.0 \pm 266.9$ & $123.6 \pm 159.6$ & .021 & .229 & .037 & .067 & 0.91 \\
\hline \multicolumn{13}{|l|}{ CS-PFP10 } \\
\hline Total & $37.8 \pm 13.1$ & $39.2 \pm 15.4$ & $1.4 \pm 12.7$ & .707 & $45.4 \pm 18.1$ & $55.7 \pm 16.0$ & $10.3 \pm 9.3$ & .003 & .252 & .017 & .025 & 1.05 \\
\hline UBS & $37.1 \pm 16.1$ & $39.2 \pm 17.0$ & $2.1 \pm 13.8$ & .610 & $46.9 \pm 15.6$ & $58.5 \pm 16.8$ & $11.6 \pm 10.2$ & .002 & .146 & .011 & .032 & 1.14 \\
\hline LBS & $33.9 \pm 10.1(n=11)$ & $33.5 \pm 16.1$ & $1.1 \pm 12.1(n=11)$ & .763 & $39.4 \pm 17.8$ & $50.7 \pm 17.3$ & $11.3 \pm 10.2$ & .003 & .383 & .019 & .032 & 1.03 \\
\hline UBS Flex & $61.0 \pm 14.1$ & $61.5 \pm 14.8$ & $0.5 \pm 17.6$ & .925 & $59.8 \pm 20.3$ & $69.4 \pm 18.5$ & $9.6 \pm 8.4$ & .002 & .868 & .261 & .107 & 0.47 \\
\hline BALC & $38.8 \pm 13.4$ & $39.8 \pm 17.2$ & $1.1 \pm 14.7$ & .804 & $46.2 \pm 20.8$ & $55.8 \pm 17.5$ & $9.7 \pm 10.1$ & .007 & .310 & .034 & .047 & 0.92 \\
\hline END & $37.2 \pm 13.4$ & $38.1 \pm 16.6$ & $0.9 \pm 13.2$ & .808 & $45.4 \pm 19.6$ & $55.2 \pm 16.7$ & $9.8 \pm 9.3$ & .004 & .244 & .020 & .029 & 1.02 \\
\hline \multicolumn{13}{|l|}{ HROOL } \\
\hline DASI & $45.8 \pm 11.1$ & $41.9 \pm 10.1$ & $-3.9 \pm 8.7$ & .146 & $49.2 \pm 9.0$ & $49.2 \pm 6.9$ & $-0.1 \pm 8.7$ & .981 & .410 & .051 & .076 & 0.84 \\
\hline MLHFO & $49.8 \pm 26.0$ & $46.5 \pm 19.7$ & $-3.3 \pm 18.6$ & .547 & $56.1 \pm 24.4$ & $33.5 \pm 22.9$ & $-22.6 \pm 18.4$ & .001 & .551 & .151 & .017 & 0.61 \\
\hline ESS & $8.5 \pm 3.5$ & $10.0 \pm 5.4$ & $1.5 \pm 4.5$ & .277 & $7.2 \pm 3.2$ & $6.2 \pm 3.5$ & $-1.0 \pm 3.4$ & .324 & .340 & .051 & .096 & 0.84 \\
\hline $\mathrm{BDI}$ & $17.4 \pm 9.1(n=11)$ & $12.9 \pm 9.0$ & $-3.5 \pm 12.0(n=11)$ & .350 & $11.5 \pm 6.8(n=11)$ & $8.9 \pm 6.2$ & $-2.1 \pm 8.7(n=11)$ & .442 & .099 & .218 & .299 & 0.52 \\
\hline
\end{tabular}

NOTE. Values expressed as mean $\pm \mathrm{SD}$ unless noted otherwise. Cohen $d=\mathrm{t} \sqrt{\frac{\mathrm{n} 1+\mathrm{n} 2}{\mathrm{n} 1^{*} \mathrm{n} 2}}$, where $t$ is test statistic for the 2-group $t$ test at post, $\mathrm{n} 1$ is sample size for control, and $\mathrm{n} 2$ is sample size for exercise groups. ${ }^{49}$

Abbreviations: ANCOVA, analysis of covariance; BALC, 10-Item Continuous Scale Physical Functional Performance Balance and Coordination domain; BDI, Beck Depression Inventory; END, 10-Item Continuous Scale Physical Functional Performance Endurance domain; ext, extension; FA, forearm; flex, flexion; L, left; LBS, 10-Item Continuous Scale Physical Functional Performance Lower-Body Strength domain; R, right; UBS, 10-Item Continuous Scale Physical Functional Performance Upper-Body Strength domain.

*Nonpooled $t$ test performed. 


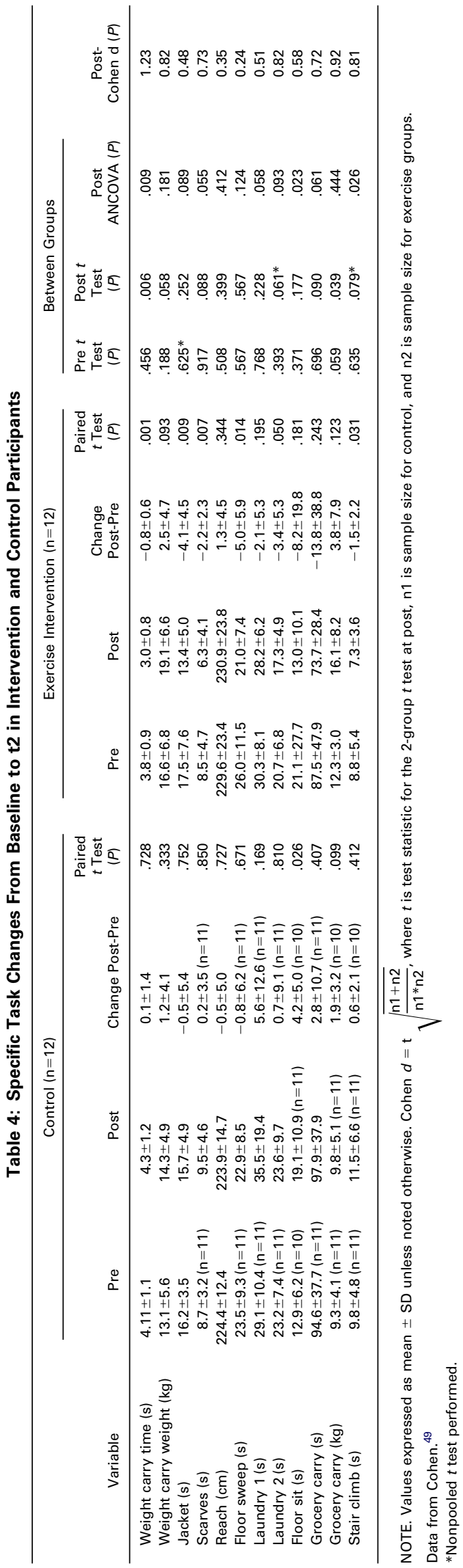

Subjective responses associated with quality of life (MLHFQ) were significantly better at $\mathrm{t} 2(P<.001)$. Responses on functional capacity (DASI) improved after 12 weeks of exercise (see table 3), but were not significant. Scores on the ESS decreased, but not significantly, suggesting that participants perceived less daytime sleepiness compared with baseline. At baseline, there also was moderate correlation between the physical subscale of the MLHFQ and CS-PFP10 total score $(r=.48 ; P<.018)$. At $\mathrm{t} 2$, changes in total CS-PFP10 scores correlated with changes in handgrip $(r=.51 ; p<.01)$ and knee extension $(r=.42 ; P<.04)$, but did not correlate significantly with subjective responses associated with the MLHFQ or DASI (not shown). Adherence was $83 \%$ and $99 \%$ for the walking sessions and resistance exercises, respectively. Mean number of steps increased by 1500 per session $(P<.001)$ during the first consecutive 8 weeks of the intervention.

\section{Attention Control Wait List Group}

In the control group, there were no significant changes for any of the outcome variables. Overall CS-PFP10 score essentially was unchanged at $\mathrm{t} 2(38 \pm 13$ vs $39 \pm 15 ; P<.70)$, and none of the 5 domain scores increased from baseline. Notably, it took significantly longer for attention control wait list group participants to perform the grocery carry, 1 of the difficult tasks, at $\mathrm{t} 2$ (see table 4).

There were clinically meaningful improvements in HRQOL, evidenced by a score change of 5 points on the MLHFQ. However, perceptions of functional capacity were lower and daytime sleepiness and depressive symptoms were higher compared with baseline (see table 3).

\section{Between Groups}

Between-group analysis showed that a decrease in time to complete most tasks was greater in exercise participants compared with the attention control wait list group (see table 3). Specifically, exercise participants performed the pot carry and laundry test 1 , as well as got up from the floor significantly more quickly, than the attention control wait list group, adjusting for covariates. Weight during the pot carry tended to be higher in the exercise group and was significantly higher for the grocery carry, but was not significant after covariate adjustments were made. There also were significant increases in total CS-PFP10 score and most domain scores compared with the attention control wait list group (see table 3). Exercise participants had significant improvements in physical function, HRQOL, and daytime sleepiness compared with the attention control wait list group (see table 3 ). The effect was moderate for most intervention variables, including the CS-PFP10, muscle strength, and HRQOL measures. Most specific tasks also had moderate effect sizes, with the exception of the floor sweep and reach test (see table 4 ).

\section{DISCUSSION}

\section{Physical Function}

Physical function, or the capacity to perform PADLs such as walking and performing household chores, ${ }^{50}$ substantially decreases in patients with $\mathrm{HF}$ as a consequence of decreased aerobic capacity and skeletal muscle myopathy. ${ }^{1-5}$ Supporting our hypothesis, the major finding from this study was that a program of combined aerobic and resistance exercise was able to significantly improve speed and ability to carry heavier amounts of weighted items that simulate physical tasks that are routinely performed by most patients with NYHA classes II and III. This study clearly showed the poor physical function experienced by most patients 
with HF and was lower in patients with NYHA class III, as anticipated. Most (92\%) participants had poor physical function at baseline, evidenced by total CS-PFP10 scores less than 48, which indicated a higher probability of experiencing loss of independency and disability. However, participants in the exercise group had significant improvements in physical function that increased from 45 (less than threshold levels) to 56 (near threshold levels), associated with independent living during a 12-week period. We also found that even complex patients with HF with a poorly functioning left ventricle and an ICD can safely derive benefit from a home-based exercise program if close monitoring and careful progression are used. The finding that muscle strength improved after a combined aerobic and resistance exercise was similar to that in previous studies. ${ }^{51-54}$ However, to our knowledge, this is the first study to document objective improvement in ability to perform PADLs after exercise in patients with HF.

Only 1 other study evaluated specific tasks included in the CS-PFP10 as an outcome measure after an exercise intervention in patients with cardiovascular disease. ${ }^{55,56}$ In that study, there were similar improvements in specific tasks, as well as CS-PFP10 total and domain scores, in older women (mean age, $72 \mathrm{y}$ ) with CAD after a 6-month resistance training program. The increase in weighted task items in older women with CAD and endurancerelated tasks were similar to our findings. For example, the mean increase in distance walked on the 6MWT was approximately $49 \mathrm{~m}$ in women with CAD compared with $55 \mathrm{~m}$ in our sample. In a previous study, we observed that aerobic exercise alone did not improve performance for the weighted items. ${ }^{57}$ These findings suggest that a combined aerobic and resistance program may have an advantage over either exercise mode alone for improving functional performance in patients with advanced cardiovascular disease, but needs further study.

Findings from this study suggest that patients with NYHA class III who had lower physical function and greater disease severity may derive benefit from exercise, although results from this small sample size should be interpreted with caution. CS-PFP10 scores also indicate that patients with NYHA class III were more vulnerable to losing independence; however, after exercise, they had significant gains in physical function that may potentially translate to decreased risk for losing independence. ${ }^{27}$ Routine evaluation that uses objective multidomain performance-based measures such as the CS-PFP10 may detect subtle clinical changes and provide time to intervene in patients with HF before functional abilities become even more compromised.

The CS-PFP10 provides information that may be used to supplement traditional exercise tests for a more comprehensive evaluation of physical function. Domain scores may help clarify the nature of self-reported functional limitations. For example, in 2 patients with similar CS-PFP10 total scores, the domain subscales might show that 1 adult has problems with the 10-Item Continuous Scale Physical Functional Performance Upper-Body Strength domain and balance, whereas the other has problems with 10-Item Continuous Scale Physical Functional Performance Lower-Body Strength domain and 10-Item Continuous Scale Physical Functional Performance Endurance domain. ${ }^{25-27}$ With this information, clinicians would be able to more closely prescribe tailored exercise, more appropriately targeting exercises to improve physical function for domainspecific areas. ${ }^{23-27}$ In a larger more diverse sample, there is potential to develop normative data by which individual CSPFP10 and domain scores may be used as benchmarks against such established values as NYHA class, $\mathrm{VO}_{2}$ peak, or other reference points.

\section{Brain Natriuretic Peptide}

The finding of increased BNP levels in some participants after exercise was documented in some, but not all, studies. ${ }^{58,59}$ The increase in BNP levels from 184 153 (range, 11-428pg/ $\mathrm{mL}$ ) to $306 \pm 269 \mathrm{pg} / \mathrm{mL}$ (range, $5-929 \mathrm{pg} / \mathrm{mL}$ ) occurred in $54 \%$ $(\mathrm{n}=13)$ of participants. In the 5 participants (2 exercise, 3 control) with BNP levels greater than $400 \mathrm{pg} / \mathrm{mL}$ at baseline, 3 increased ( 1 exercise, 2 control) and 2 decreased (1 control, 1 exercise), indicating that factors other than exercise contributed to the higher BNP levels. ${ }^{59}$ Previous studies showed that BNP level greater than $324 \mathrm{pg} / \mathrm{mL}$ was associated with a risk ratio of 8.8 for adverse cardiac events. ${ }^{60}$ However, variability in BNP level is well documented, and these results must be interpreted with caution. 61

\section{Health-Related Quality of Life}

The few exercise studies that included HRQOL as an outcome measure had limited and inconsistent findings. For example, Belardinelli et $\mathrm{al}^{62}$ found that changes in HRQOL paralleled changes in $\mathrm{VO}_{2}$ peak, whereas other studies found no association between HRQOL and aerobic capacity. ${ }^{63,64}$ A recent meta-analysis examining the effects of exercise training on HRQOL in patients with $\mathrm{HF}$ found only 1 study that included a significant positive correlation between change in aerobic capacity and change in HRQOL. ${ }^{65}$ In the present study, we found that despite significant improvement in HRQOL in exercise participants, there was no relationship to subjective or objective measures of physical function at 2 . Participants in the attention control wait list group experienced clinically meaningful improvement in HRQOL. Reasons may have included the attention from the research team during the placebo exercise sessions. In addition, attention control wait list participants may have perceived improvement in physical function from involvement in the placebo exercise, which in turn may have positively influenced HRQOL. The knowledge that they would receive the same exercise equipment and instruction at the end of the study also may have improved HRQOL in the attention control wait list group. Finally, our findings also suggest that exercise may provide an inexpensive and readily available treatment option that may improve daytime sleepiness and depressive symptoms in patients with HF, a major contributing factor for poor HRQOL in this patient population. ${ }^{66,67}$

\section{Study Limitations}

This study had several limitations that may influence generalization of results. First, sample size was small and prevented the use of tests to evaluate change across time between groups in a comprehensive procedure. In addition, data were collected primarily from 1 academic center and may have lacked general representation of patients with HF. Data collectors were not blinded to group allocation, which introduces the possibility of bias. A standard protocol was adhered to during administration of the CS-PFP10, and enough time had passed that familiarity was not a significant factor concerning study outcomes. Despite randomization, there was a greater number of patients with diabetes in the attention control wait list group, which may have influenced physical function outcomes. Therefore, results should be interpreted with caution. Enrolling patients with HF is difficult, shown by only 21 of the eligible 152 responding to recruitment despite intensive recruitment efforts on the part of the investigators. The ability and practicality of providing this type of exercise intervention remains uncertain in a larger more diverse group of patients with HF.

\section{CONCLUSIONS}

Findings from this study suggest that complex patients with $\mathrm{HF}$ are able and willing to perform exercise safely and at a level that 
improves the ability to perform measured tasks. The study also showed the feasibility and acceptability by patients of a homebased aerobic and resistance exercise program that may be used to design future interventions. A combined regimen of aerobic and resistance exercise may be the most advantageous for patients with HF in terms of optimizing physical function, maximizing independence, and enhancing HRQOL, but needs further study in a larger more diverse $\mathrm{HF}$ population.

\section{References}

1. Aronow WS. Epidemiology, pathophysiology, prognosis, and treatment of systolic and diastolic heart failure. Cardiol Rev 2006; $14: 108-24$.

2. Niebauer J. Effects of exercise training on inflammatory markers in patients with heart failure. Heart Fail Rev 2008;13:39-49.

3. Braith RW, Beck DT. Resistance exercise: training adaptations and developing a safe exercise prescription. Heart Fail Rev 2008; 13:69-79.

4. McKelvie RS. Exercise training in patients with heart failure: clinical outcomes, safety, and indications. Heart Fail Rev 2008; 13:3-11.

5. Sullivan MJ, Higginbotham MB, Cobb FR. Exercise training in patients with severe left ventricular dysfunction: hemodynamic and metabolic effects. Circulation 1988;78:506-15.

6. O'Connor CM, Whellan DJ, Lee KL, et al; HF-ACTION Investigators. Efficacy and safety of exercise training in patients with chronic heart failure: HF-ACTION randomized controlled trial. JAMA 2009;301:1439-50.

7. Volaklis KA, Tokmakidis SP. Resistance exercise training in patients with heart failure. Sports Med 2005;35:1085-103.

8. Cheetham C, Green D, Collis J, Dembo L, O'Driscoll G. Effect of aerobic and resistance exercise on central hemodynamic responses in severe chronic heart failure. J Appl Physiol 2002;93:175-80.

9. Meyer K, Hajric R, Westbrook S, et al. Hemodynamic responses during leg press exercise in patients with chronic congestive heart failure. Am J Cardiol 1999;83:1537-43.

10. Williams MA, Haskell WL, Ades PA, et al; American Heart Association Council on Clinical Cardiology; American Heart Association Council on Nutrition, Physical Activity, and Metabolism. Resistance exercise in individuals with and without cardiovascular disease: 2007 update: a scientific statement from the American Heart Association Council on Clinical Cardiology and Council on Nutrition, Physical Activity, and Metabolism. Circulation 2007;116:572-84.

11. Afilalo J, Karunananthan S, Eisenberg MJ, Alexander KP, Bergman $\mathrm{H}$. Role of frailty in patients with cardiovascular disease. Am J Cardiol 2009;103:1616-21.

12. Chiarantini D, Volpato S, Sioulis F, et al. Lower extremity performance measures predict long-term prognosis in older patients hospitalized for heart failure. J Card Fail 2010;16:390-5.

13. Applegate WB, Blass JP, Williams TF. Instruments for the functional assessment of older patients. N Engl J Med 1990;322: 1207-14.

14. Reuben DB, Valle LA, Hays RD, Siu AL. Measuring physical function in community-dwelling older persons: a comparison of self-administered, interviewer-administered, and performancebased measures. J Am Geriatr Soc 1995;43:17-23.

15. Studenski S, Perera S, Wallace D, et al. Physical performance measures in the clinical setting. J Am Geriatr Soc 2003;51:314-22.

16. Guralnik JM, Ferrucci L. Assessing the building blocks of function: utilizing measures of functional limitation. Am J Prev Med 2003;25(3 Suppl 2):112-21.

17. Inouye SK, Paduzzi PN, Robison JT, et al. Importance of functional measures in predicting mortality among older hospitalized patients. JAMA 1998;279:1187-93.
18. Purser JL, Kuchibhatla MN, Fillenbaum GG, Harding T, Peterson $\mathrm{ED}$, Alexander KP. Identifying frailty in hospitalized older adults with significant coronary artery disease. J Am Geriatr Soc 2006; 54:1674-81.

19. Afilalo J, Eisenberg MJ, Morin JF, et al. Gait speed as an incremental predictor of mortality and major morbidity in elderly patients undergoing cardiac surgery. J Am Coll Cardiol 2010;56: 1668-76.

20. Lee DH, Buth KJ, Martin BJ, Yip AM, Hirsch GM. Frail patients are at increased risk for mortality and prolonged institutional care after cardiac surgery. Circulation 2010;121:973-8.

21. Wilson JR, Hanamanthu S, Chomsky DB, Davis SF. Relationship between exertional symptoms and functional capacity in patients with heart failure. J Am Coll Cardiol 1999;33:1943-7.

22. Wilson J, Rayos G, Yeoh T, Gothard P, Bak K. Dissociation between exertional symptoms and circulatory function in patients with heart failure. Circulation 1995;92:47-53.

23. Guralnik JM, Ferrucci L, Simonsick EM, Salive ME, Wallace RB. Lower-extremity function in persons over the age of 70 years as a predictor of subsequent disability. N Engl J Med 1995;332:556-61.

24. Bergman H, Ferrucci L, Guralnik J, et al. Frailty: an emerging research and clinical paradigm-issues and controversies. J Gerontol A Biol Sci Med Sci 2007;62:731-7.

25. Cress ME, Buchner DM, Questad KA, Esselman PC, deLateur BJ, Schwartz RS. Continuous-scale physical functional performance in healthy older adults: a validation study. Arch Phys Med Rehabil 1996;77:1243-50.

26. Cress ME, Petrella JK, Moore TL, Schenkman ML. ContinuousScale Physical Functional Performance test: validity, reliability, and sensitivity of data for the short version. Phys Ther 2005;85: 323-35.

27. Cress ME, Meyer M. Maximal voluntary and functional performance levels needed for independence in adults aged 65 to 97 years. Phys Ther 2003;83:37-48.

28. Borg G. Psychophysical bases of perceived exertion. Med Sci Sports Exerc 1982;14:377-81.

29. Guyatt GH, Sullivan MJ, Thompson PJ, et al. The 6-minute walk: a new measure of exercise capacity in patients with chronic heart failure. CMAJ 1985;132:919-23.

30. Gary R, Sueta CA, Rosenberg B, Cheek D. The Six-Minute Walk Test as a measure of exercise effectiveness in older women with diastolic heart failure. J Cardiopulm Rehabil 2004;24:264-8.

31. Bittner V, Weiner D, Yusuf S, et al. Prediction of mortality and morbidity with a Six-Minute Walk Test in patients with left ventricular function. JAMA 1993;270:1202-7.

32. Shyam Kumar AJ, Parmar V, Ahmed S, Kar S, Harper WM. A study of grip endurance and strength in different elbow positions. J Orthop Traumatol 2008;9:209-11.

33. Schaubert KL, Bohannon RW. Reliability and validity of three strength measures obtained from community-dwelling elderly persons. J Strength Cond Res 2005;19:717-20.

34. Wang CY, Olson SL, Protas EJ. Test-retest strength reliability: hand-held dynamometry in community-dwelling elderly fallers. Arch Phys Med Rehabil 2002;83:811-5.

35. Wenger NK. Quality of life: can it and should it be assessed in patients with heart failure? Cardiology 1989;76:391-8.

36. Rector TS, Kubo SH, Cohn JN. Patients' self-assessment of their congestive heart failure. Heart Fail 1987;October/November:198209.

37. Middel B, Bouma J, deJongste M, et al. Psychometric properties of the Living With Heart Failure Questionnaire.(MLHF-Q). Clin Rehabil 2001;15:489-500.

38. Hlatky MA, Boineau RE, Higginbotham MB, et al. A brief selfadministered questionnaire to determine functional capacity (The Duke Activity Status Index). Am J Cardiol 1989;64:651-4. 
39. Nelson CL, Herndon JE, Mark DB, Pryor DB, Califf RM, Hlatky MA. Relation of clinical and angiographic factors to functional capacity as measured by the Duke Activity Status Index. Am J Cardiol 1991;68:973-5.

40. Johns MW. A new method for measuring daytime sleepiness: the Epworth Sleepiness Scale. Sleep 1991;4:540-5.

41. Luther SA, McCullough PA, Havranek EP, et al. The relationship between B-type natriuretic peptide and health status in patients with heart failure. J Card Fail 2005;11:414-21.

42. Anderson KM. Clinical uses of brain natriuretic peptide in diagnosing and managing heart failure. J Am Acad Nurse Pract 2008; 20:305-10

43. Charlson ME, Pompei P, Ales KL, MacKenzie CR. A new method of classifying prognostic comorbidity in longitudinal studies: development and validation. J Chronic Dis 1987;40:373-83.

44. Karvonen M, Kentala K Mustalo O. The effects of training on heart rate: a longitudinal study. Ann Med Exp Biol Fenn 1957; 35:307-15.

45. Heart Failure Society of America. Executive summary: HFSA 2006 Comprehensive Heart Failure Practice Guideline. J Card Fail 2006;12:10-38.

46. Evangelista LS, Dracup K, Erickson V, McCarthy WJ, Hamilton MA, Fonarow GC. Validity of pedometers for measuring exercise adherence in heart failure patients. J Card Fail 2005;11:366-71.

47. Tudor-Locke C, Lutes L. Why do pedometers work? A reflection upon the factors related to successfully increasing physical activity. Sports Med 2009;39:981-93.

48. Bravata DM, Smith-Spangler C, Sundaram V, Gienger AL, Lin N, Lewis R. Using pedometers to increase physical activity and improve health: a systematic review. JAMA 2007;298:2296-304.

49. Cohen J. Statistical power analysis for the behavioral sciences. 2nd ed. New York: Psychology Pr; 1988. p 67.

50. Caspersen CJ. Physical activity epidemiology: concepts, methods, and applications to exercise science. Exerc Sport Sci Rev 1989; 17:423-73.

51. Beckers PJ, Denollet J, Possemiers NM, Wuyts FL, Vrints CJ, Conraads VM. Combined endurance-resistance vs. endurance training in patients with chronic heart failure: a prospective randomized study. Eur Heart J 2008;29:1858-66.

52. Delagardelle C, Feiereisen P, Autier P, Shita R, Krecke R, Beissel J. Strength/endurance training versus endurance training in congestive heart failure. Med Sci Sports Exerc 2002;34:1868-72.

53. Mandic S, Tymchak W, Kim D, et al. Effects of aerobic or aerobic and resistance training on cardiorespiratory and skeletal muscle function in heart failure: a randomized controlled pilot trial. Clin Rehabil 2009;23:207-16.

54. Haykowsky M, Vonder Muhll I, Ezekowitz J, Armstrong P. Supervised exercise training improves aerobic capacity and muscle strength in older women with heart failure. Can J Cardiol 2005; 21:1277-80

55. Brochu M, Savage P, Lee M, et al. Effects of resistance training on physical function in older disabled women with coronary heart disease. J Appl Physiol 2002;92:672-8.
56. Ades PA, Savage PD, Cress ME, Brochu M, Lee NM, Poehlman ET. Resistance training on physical performance in disabled older female cardiac patients. Med Sci Sports Exerc 2003;35:1265-70.

57. Gary R, Cress ME, Hayes DM, Moore TL. Performance of physical activities of daily living among New York Heart Association class II and III heart failure patients. Presented at: 11th Annual Heart Failure Society of America, September 16-19, 2007, Washington (DC)

58. Maria Sarullo F, Gristina T, Brusca I, et al. Effect of physical training on exercise capacity, gas exchange and $\mathrm{N}$-terminal probrain natriuretic peptide levels in patients with chronic heart failure. Eur J Cardiovasc Prev Rehabil 2006;13:812-7.

59. Pascual-Figal DA, Penafiel P, de la Morena G, et al. Relation of B-type natriuretic peptide levels before and after exercise and functional capacity in patients with idiopathic dilated cardiomyopathy. Am J Cardiol 2007;99:1279-83.

60. Koc M, Bozkurt A, Acarturk E, Sahin DY, Unal I. Usefulness of $\mathrm{N}$-terminal pro-B-type natriuretic peptide increase with exercise for predicting cardiovascular mortality in patients with heart failure. Am J Cardiol 2008;101:1157-62.

61. O'Hanlon R, O'Shea P, Ledwidge M, et al. The biologic variability of B-type natriuretic peptide and N-terminal pro-B-type natriuretic peptide in stable heart failure patients. J Card Fail 2007;13: 50-5.

62. Belardinelli R, Georgiou D, Cianci G, Purcaro A. Effects of exercise training on left ventricular filling at rest and during exercise in patients with ischemic cardiomyopathy and severe left ventricular systolic dysfunction. Am Heart J 1996;132(1 Pt 1): 61-70.

63. Oka RK, De Marco T, Haskell WL, et al. Impact of a home-based walking and resistance training program on quality of life in patients with heart failure. Am J Cardiol 2000;85:365-9.

64. Wielenga RP, Erdman RA, Huisveld IA, et al. Effect of exercise training on quality of life in patients with chronic heart failure. J Psychosom Res 1998;45:459-64.

65. van Tol BA, Huijsmans RJ, Kroon DW, Schothorst M, Kwakkel G. Effects of exercise training on cardiac performance, exercise capacity and quality of life in patients with heart failure: a metaanalysis. Eur J Heart Fail 2006;8:841-50.

66. Johansson P, Dahlstrom U, Brostrom A. Factors and interventions influencing health-related quality of life in patients with heart failure: a review of the literature. Eur J Cardiovasc Nurs 2006;5: 5-15.

67. Redeker NS, Hilkert R. Sleep and quality of life in stable heart failure. J Card Fail 2005;11:700-4.

\section{Suppliers}

a. Polar Electro Inc, 1111 Marcus Ave, Ste M15, Lake Success, NY $11042-1034$

b. Sammons-Preston, 1000 Remington Blvd, Ste 210, Bolingbrook, IL 60440-5117.

c. Biosite Inc, 9975 Summers Ridge Rd, San Diego, CA 92121.

d. The Hygenic Corp, 1245 Home Ave, Akron, OH 44310.

e. Omron Healthcare Inc, 1200 Lakeside Dr, Bannockburn, IL 60015.

f. SPSS Inc 233 S Wacker Dr, 11th Fl, Chicago, IL 60606. 